\title{
Dendro-Dendritic Interactions between Motion-Sensitive Large-Field Neurons in the Fly
}

\author{
Juergen Haag and Alexander Borst \\ Department of Environmental Science, Policy, and Management, Division of Insect Biology, University of California, \\ Berkeley, Berkeley, California 94720
}

\begin{abstract}
For visual course control, flies rely on a set of motion-sensitive neurons called lobula plate tangential cells (LPTCs). Among these cells, the so-called $\mathrm{CH}$ (centrifugal horizontal) cells shape by their inhibitory action the receptive field properties of other LPTCs called FD (figure detection) cells specialized for figureground discrimination based on relative motion. Studying the ipsilateral input circuitry of $\mathrm{CH}$ cells by means of dual-electrode and combined electrical-optical recordings, we find that $\mathrm{CH}$
\end{abstract}

cells receive graded input from HS (large-field horizontal system) cells via dendro-dendritic electrical synapses. This particular wiring scheme leads to a spatial blur of the motion image on the $\mathrm{CH}$ cell dendrite, and, after inhibiting FD cells, to an enhancement of motion contrast. This could be crucial for enabling FD cells to discriminate object from self motion.

Key words: calcium; dendrite; electrophysiology; insect; motion detection; vision
Image segmentation into objects and background is of prime importance for visual orientation and can rely on several cues. Fast-flying animals, such as dipteran flies, have shown to use motion cues for this task. When flying tethered in a well defined environment, i.e., an arena consisting of a foreground figure and a background pattern, flies consistently exert torque toward the figure whenever the figure moves relative to the background (Heisenberg and Wolf, 1979; Reichardt and Poggio, 1979; Egelhaaf 1985a; Kimmerle and Egelhaaf, 2000a). Analysis of the neural circuitry underlying this motion-based figure-ground discrimination revealed a specific class of neurons termed FD (figure detection) cells (Egelhaaf, 1985b), which become selectively activated by motion of small objects in front of an extended background. This response selectivity vanished after laser ablation of the so-called vCH (ventral centrifugal horizontal) cell (Warzecha et al., 1993), an inhibitory GABAergic neuron (Meyer et al., 1986) with output synapses located within the dendrite (Gauck et al., 1997).

FD and $\mathrm{CH}$ cells belong to a set of $\sim 60$ individually identifiable motion-sensitive neurons called lobula plate tangential cells (LPTCs) (Hausen et al., 1980; Hausen, 1982, 1984; Hengstenberg et al., 1982; Borst and Haag, 1996; Haag and Borst, 2001). Among the LPTCs, HS (large-field horizontal system) and VS (vertical system) cells are thought to be the major output elements conveying information about large-field horizontal and vertical image motion onto descending neurons, which ultimately control motor neurons for locomotion or head movements (Gronenberg and Strausfeld, 1990; Gilbert et al., 1995; Chan et al., 1998). Whereas most LPTCs are thought to spatially integrate the

\footnotetext{
Received Nov. 5, 2001; revised Jan. 16, 2002; accepted Feb. 4, 2002.

This work was supported by National Institutes of Health Grant 1RO1MH6159801 to A.B. We are grateful to Dr. Dierk Reiff for discussions and carefully reading this manuscript.

Correspondence should be addressed to Alexander Borst at his present address: Max-Planck-Institute of Neurobiology, Department of Systems and Computational Neurobiology, Am Klopferspitz 18a, D-82152 Martinsried, Germany. E-mail: borst@neuro.mpg.de.

Copyright (C) 2002 Society for Neuroscience $\quad 0270-6474 / 02 / 223227-07 \$ 15.00 / 0$
}

output signals of many thousands of columnar, local motionsensitive neurons on their large dendrite in the lobula plate, the origin of motion sensitivity in $\mathrm{CH}$ cells remains controversial. Based on the observations of anatomical structures called "blebs" in their large lobula plate arborizations, $\mathrm{CH}$ cells were first concluded to be solely presynaptic in the lobula plate and, therefore, were christened "centrifugal horizontal" (Eckert and Dvorak, 1983). CH cells were thought to inherit motion sensitivity from HS cells via their ramifications in the protocerebrum. However, after local motion stimulation, local calcium accumulation was observed in the dendrites of $\mathrm{CH}$ cells (Egelhaaf et al., 1993). This fact is incompatible with the assumption of $\mathrm{CH}$ cells being postsynaptic to the axon terminal of HS cells only, in which all of the motion information is spatially integrated, and any positional information is completely lost.

On the other hand, precise knowledge about the input circuitry of $\mathrm{CH}$ cells and exactly where on the extended branches of the neurons these cells make contact seems to be crucial for understanding the essence of the neural mechanism underlying figureground discrimination: one cell inhibiting another does not necessarily tune the latter sensitive to small moving objects. To decide from which neurons of the lobula plate $\mathrm{CH}$ cells receive their motion-sensitive input and where this input converges onto their extended ramifications, i.e., lobula plate or protocerebrum, we, therefore, studied the connectivity between $\mathrm{CH}$ cells and other motion-sensitive neurons with similar visual response properties, i.e., HS cells. Using dual intracellular and combined optical-electrical recording techniques, we show in the following that $\mathrm{CH}$ cells receive ipsilateral visual motion information via electrical dendro-dendritic synapses from HS cells. As will be discussed in detail, this particular wiring cannot only resolve a number of puzzling facts about $\mathrm{CH}$ cells collected in the past but seems also appropriate for the function of $\mathrm{CH}$ cells in the context of figureground discrimination.

\section{MATERIALS AND METHODS}

Preparation and set up. Female blowflies (Calliphora vicina) were briefly anesthetized with $\mathrm{CO}_{2}$ and mounted ventral side up with wax on a small preparation platform. The head capsule was opened from behind; the 
trachea and airsacs that normally cover the lobula plate were removed. To eliminate movements of the brain caused by peristaltic contractions of the esophagus, the proboscis of the animal was cut away, and the gut was pulled out. The fly was then mounted on a heavy recording table. Visual stimuli were produced by an arc lamp, the image of which was projected onto a screen $(10 \times 8 \mathrm{~cm})$ positioned $10.5 \mathrm{~cm}$ below the fly. The square wave grating had a spatial wavelength of $12^{\circ}$, a mean luminance of 17.7 $\mathrm{cd} / \mathrm{m}^{2}$, and a contrast of $92 \%$. The fly brain was viewed from behind through a microscope (Axiotech vario 100HD; Zeiss, Oberkochen, Germany).

Electrical recording. Electrodes were pulled on a Brown-Flaming micropipette puller (P-97) using thin-wall glass capillaries with an outer diameter of $1 \mathrm{~mm}$ (GC100TF-10; Clark Electromedical Instruments, Pangbourne, UK). The tip of the electrode was filled with $8.8 \mathrm{~mm}$ Calcium Green (Ca-Green-1 hexapotassium salt; Molecular Probes, Eugene, OR), $10 \mathrm{~mm}$ Alexa 568 (Alexa Fluor 568 hydrazide; Molecular Probes), or $8.8 \mathrm{~mm}$ Calcium Orange (Ca-Orange tetrapotassium salt; Molecular Probes). The shaft of the electrode was filled with a solution containing $2 \mathrm{M} \mathrm{KAc}$ plus $0.5 \mathrm{M} \mathrm{KCl}$. Electrodes had resistances of $\sim 25$ $\mathrm{M} \Omega$. An SEL10 amplifier (NPI Electronics, Tamm, Germany) was used in the bridge mode. For the experiments with dual intracellular recordings a second SEL10 amplifier was used. All recordings were made from the axons of the cells. For data analysis, the output signal of the amplifier was fed to a Pentium III personal computer via an 12 bit analog-to-digital converter (DAS-1602; ComputerBoards, Middleboro, MA) at a sampling rate of $5 \mathrm{kHz}$.

Optical recording. We used a Zeiss UD $20 \times, 0.57$ numerical aperture (NA) air objective or an Epiplan $40 \times, 0.75$ NA water immersion lens and a CCD camera (equipped with an EEV chip, $1024 \times 512$ pixel; PXL; PhotoMetrics Inc., Huntington Beach, CA) connected to a Power Macintosh (Apple Computers, Cupertino, CA). The first frame of each image series was taken as a reference and subtracted from each following image. This resulted in a series of difference images $(\Delta F)$, which were subsequently divided by the reference frame $(\Delta F / F)$. For calcium imaging, we used Calcium Green (Molecular Probes) with the Zeiss filter set \#9 [bandpass (BP), 450-490 nm; dichroic mirror (DM), $510 \mathrm{~nm}$; longpass (LP), $520 \mathrm{~nm}$ ] and Calcium Orange (Molecular Probes) with the Zeiss filter set \#15 (BP, 546 nm; DM, 580 nm; LP, $590 \mathrm{~nm}$ ). To visualize neurons with Alexa 568 (Molecular Probes), we used the same filter set.

\section{RESULTS}

As mentioned in the introductory remarks, $\mathrm{HS}$ and $\mathrm{CH}$ cells share many visual response properties: like $\mathrm{CH}$ cells, $\mathrm{HS}$ cells are excited by horizontal front-to-back motion and respond to motion stimuli in a graded way (Eckert and Dvorak, 1983). To investigate whether this response similarity is based on direct connectivity, HS and $\mathrm{CH}$ cells located in the same hemisphere were recorded simultaneously by means of intracellular electrodes (Fig. 1). When the pattern moved in the preferred (PD) and null direction (ND) of the cells, both neurons exhibited similar response characteristics (Fig. 1a), including high-amplitude EPSPs during PD motion (Haag and Borst, 2001). In Figure 1b, the HS EPSPs were used to trigger the averaging of $\mathrm{CH}$ signals. Both averages revealed a synchronous rise with almost no delay. To investigate whether these EPSPs in the CH cell are elicited by HS EPSPs or arise in parallel, HS cells were stimulated to produce largeamplitude action potentials (Hengstenberg, 1977; Haag et al., 1997) by release from hyperpolarizing current injection (Fig. $2 c$, inset). Triggering $\mathrm{CH}$ cell signals by these HS cell rebound spikes did not reveal any distinct signals in $\mathrm{CH}$ cells (Fig. 1c). We conclude that $\mathrm{CH}$ and $\mathrm{HS}$ cells receive a common input causing the large EPSPs in parallel. Thus, either the cells are not connected with each other or the connection does not allow fast signals to travel across. To decide between these alternatives, we injected current of either polarity into one of the cells and measured the membrane voltage in the other. Figure $1 d$ shows that $\mathrm{HS}$ cells and $\mathrm{CH}$ cells followed with their membrane potential when current was injected into the partner cell. The currentvoltage relationship of the $\mathrm{HS}-\mathrm{CH}$ cell coupling as shown in
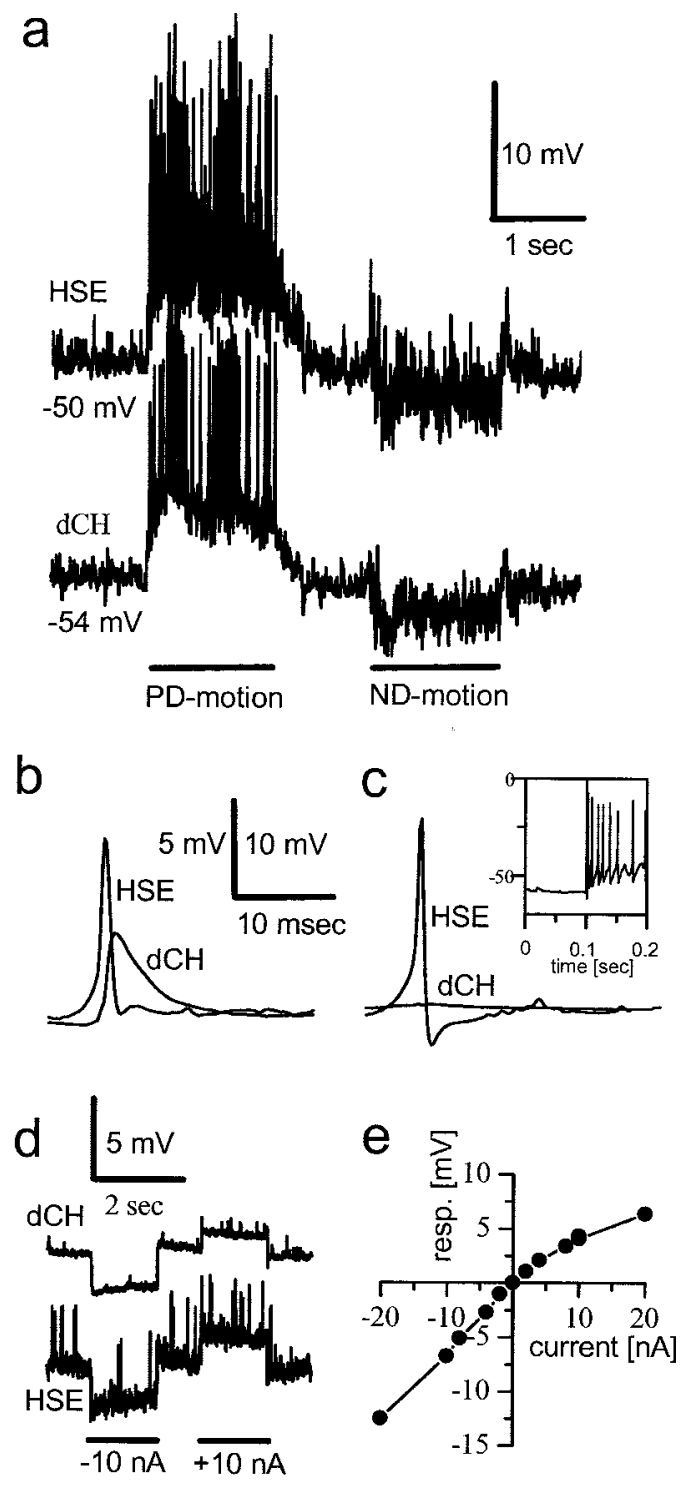

Figure 1. Dual intracellular recordings from $\mathrm{HS}$ and $\mathrm{CH}$ cells. $a, \mathrm{Re}-$ sponses to visual motion in front of both eyes along the PD and ND of the cells. $b$, Simultaneously occurring large-amplitude EPSPs in $\mathrm{HS}$ and $\mathrm{CH}$ cells. $c$, Rebound action potentials elicited in an HSE cell are not transmitted to the $\mathrm{dCH}$ cell. $d$, Response of a dCH cell to current injection into the ipsilateral HSE cell and vice versa. $e$, Current-voltage relationship between an HSN and a dCH cell. Current was injected into the HSN cell, and voltage response was measured in the $\mathrm{dCH}$ cell.

Figure $1 e$ was fairly linear after a slight outward rectification that was observed previously for the intrinsic current-voltage relationship of HS cells (Borst and Haag, 1996). The amplitude of membrane potential change in the follower cell was $\sim 10-15 \%$ of the membrane potential change recorded in the cell in which the current was directly injected. In summary, $\mathrm{HS}$ and $\mathrm{CH}$ cells are coupled to each other in a symmetrical way, with current of either polarity influencing the other cell. However, the coupling between both cells does not allow fast signals to cross between them, paralleling the previous finding that the frequency-dependent amplification is seen solely in HS cells but not in $\mathrm{CH}$ cells (Haag and Borst, 1996). Because the fine dendritic branchlets in $\mathrm{CH}$ cells do not allow for electrical recording, we cannot decide at present whether the coupling itself is slow or whether fast signals 

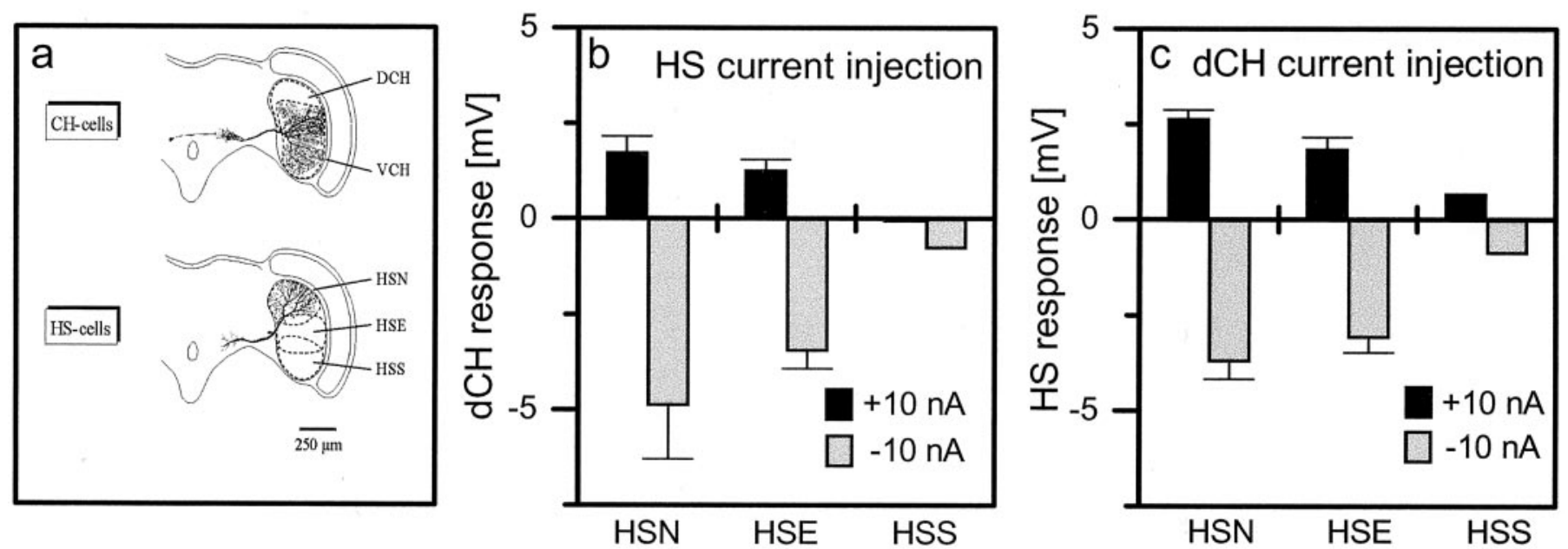

Figure 2. Strength of connectivity between various pairs of $\mathrm{HS}$ and $\mathrm{CH}$ cells. $a$, Location of $\mathrm{HS}$ and $\mathrm{CH}$ cell dendrites within the lobula plate (modified from Borst and Haag, 1996). $b$, Current of either $+10 \mathrm{nA}$ (black bars) or $-10 \mathrm{nA}$ (gray bars) amplitude was injected into HS cells, and membrane voltage was measured in the $\mathrm{dCH}$ cell. $c$, Current was injected into the $\mathrm{dCH}$ cell, and membrane voltage was measured in HS cells. Data represent the mean \pm SEM of four (HSN), five (HSE), and one (HSS) experiment.
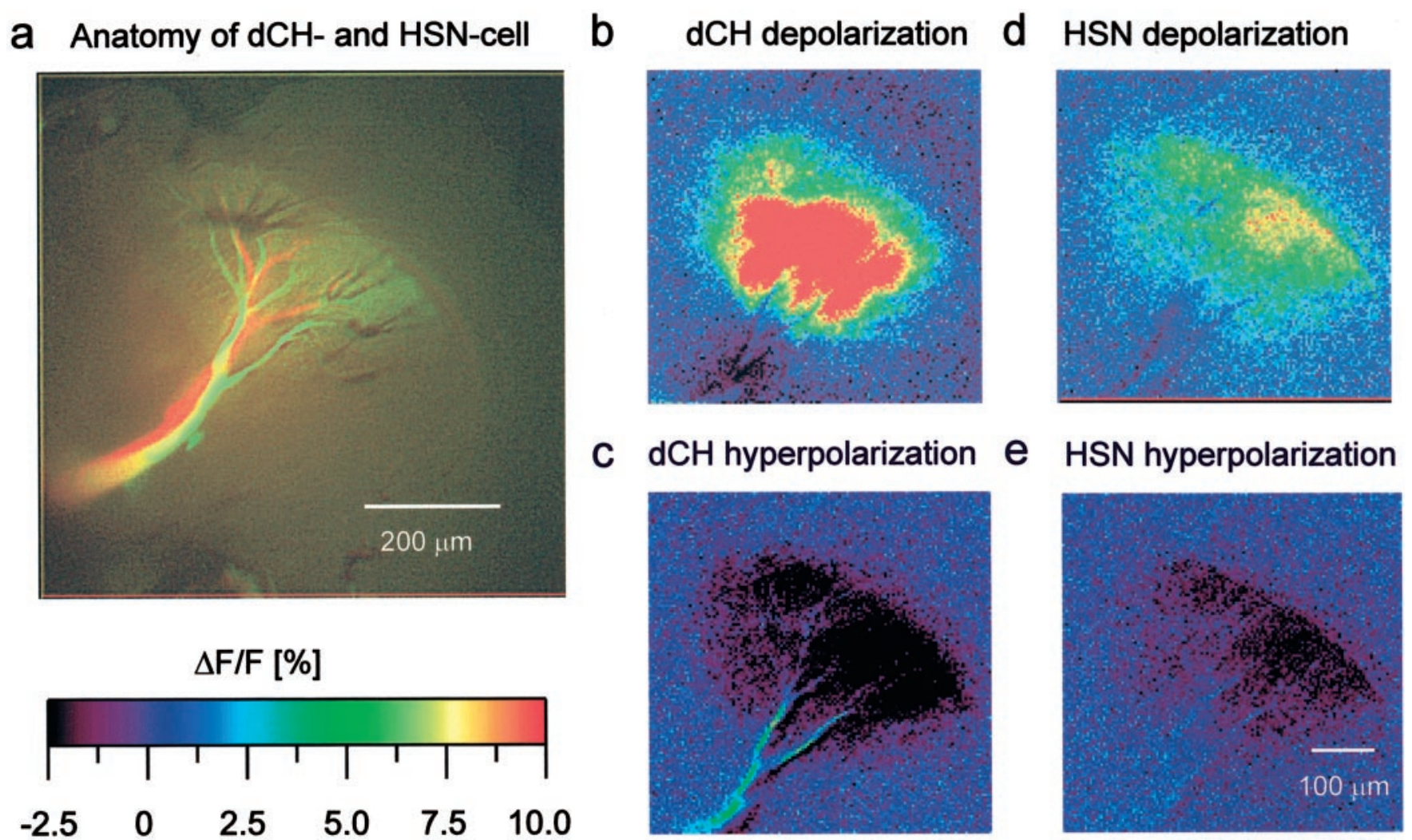

\section{$\begin{array}{llllll}-2.5 & 0 & 2.5 & 5.0 & 7.5 & 10.0\end{array}$}

Figure 3. Optical imaging of connectivity between HS and CH cells. $a$, Anatomy of both cells (lens, Epiplan $10 \times, 0.20 \mathrm{NA}$ ). $b-e$, False-color images of relative change of fluorescence $(\Delta F / F)$ occurring in the $\mathrm{dCH}$ cell under various stimulus conditions $(b, c$, direct current injection into the dCH cell; $d, e$, current injection into the HSN cell). The $\mathrm{dCH}$ cell was filled with Calcium Green, and the HSN cell was filled with Alexa (lens, Zeiss UC 20×, $0.57 \mathrm{NA}$ ).

as measured in the axon are strongly attenuated because of the low-pass filtering by the dendrite of the cell.

There exist three HS and two $\mathrm{CH}$ cells per lobula plate, which cover different regions (Fig. 2a). Using again dual-electrode intracellular recording, we investigated the coupling between all combinations of $\mathrm{HS}$ and $\mathrm{CH}$ cells. The results (Fig. 2b,c) are qualitatively identical to the experiment described above: for all pairs, we found a bidirectional coupling with hyperpolarizing current having larger effects than depolarizing current. However, the absolute coupling strength depended on the exact pair that was investigated. The influence on the dorsal centrifugal horizontal $(\mathrm{dCH})$ cell was strongest when current was injected into the horizontal system north (HSN) cells and decreased over HSE (horizontal system equatorial) to HSS (horizontal system south) 


\section{a Anatomy}

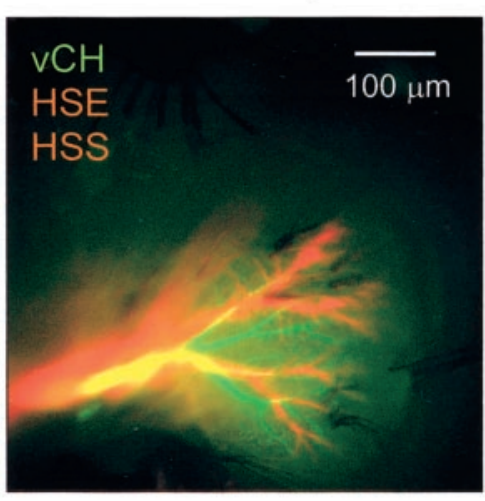

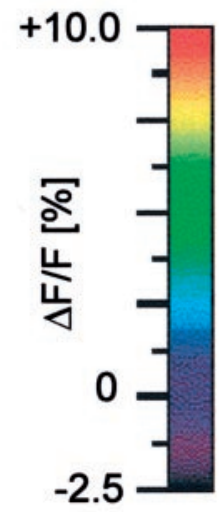

b Depolarization of $\mathrm{vCH}$

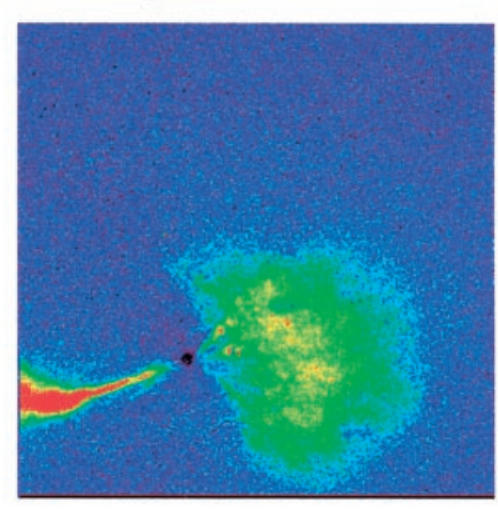

\section{Depolarization of HSE}

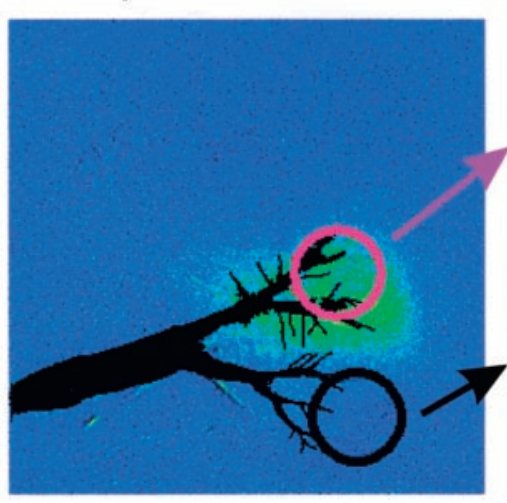

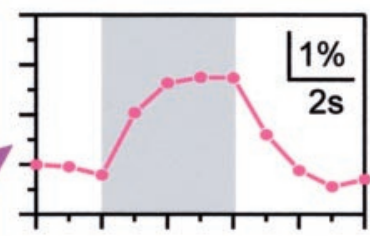

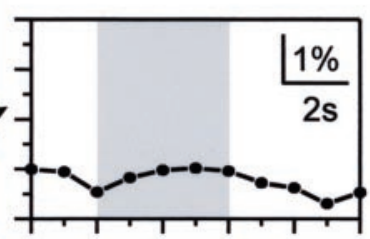

d Depolarization of HSS

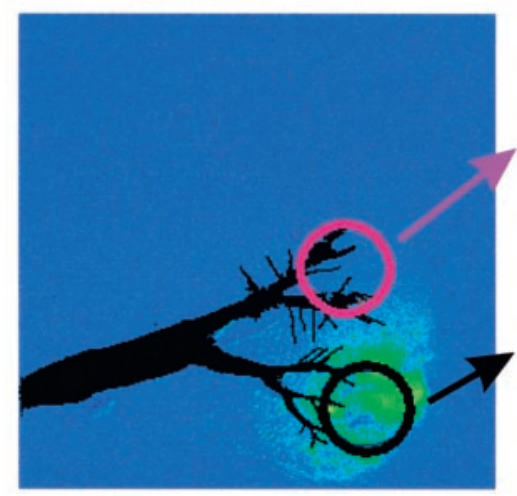

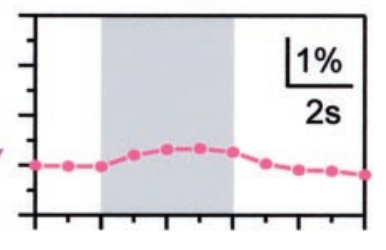

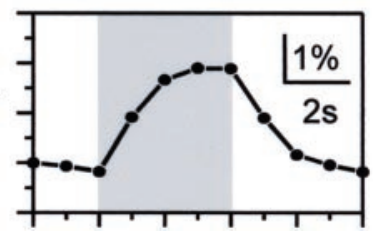

Figure 4. Dendrotopic calcium accumulation in a vCH cell. $a$, Anatomy of a vCH cell filled with Calcium Green and an HSE and an HSS cell both filled with the red fluorescent dye Alexa. $b-d$, False-color images of the fluorescence change in the vCH cell. In $c$ and $d$, the outlines of the HSE and HSS cells are superimposed in black. Also shown are the time courses of the relative change of fluorescence within the areas outlined in $c$ and $d$ during injection of depolarizing current into the HSE $(c)$ and into the HSS $(d)$ cell (lens, Zeiss UC $20 \times, 0.57$ NA).

cells (Fig. 2b). The same held true when current was injected into the $\mathrm{dCH}$ cell and the influence onto the HS cells was considered (Fig. 2c). The opposite gradation was observed for the coupling of vCH cells and HS cells: here the coupling fell off from HSS over HSE to HSN cells (data not shown). Importantly, the coupling strength between all cell pairs investigated seemed to follow the amount of overlap between the dendrites of the respective cells (Fig. 2a), suggesting that $\mathrm{HS}$ and $\mathrm{CH}$ cells are connected via their dendrites.

To test this hypothesis, we used optical recording techniques (Borst and Egelhaaf, 1992; Single and Borst, 1998). As was shown previously, the calcium signal in $\mathrm{HS}$ and $\mathrm{CH}$ cells follows the membrane potential in a rather linear way (Haag and Borst, 2000), thus making calcium a feasible indicator of activity within these cells. To see whether calcium imaging can be used to visualize connectivity, we filled one cell with a calcium indicator and subsequently recorded from the other cell electrically. Figure 3 shows the results of such an experiment. Figure $3 a$ displays the anatomy of both cells, a dCH cell filled with the calcium sensor Calcium Green (Molecular Probes) and an HSN cell loaded with the red fluorescent dye Alexa 568 (Molecular Probes) for anatomical identification. The injection of $10 \mathrm{nA}$ depolarizing current into the axon of the $\mathrm{dCH}$ cell resulted in a strong fluorescence increase in all lobula plate branches (Fig. 3b), and injection of $10 \mathrm{nA}$ hyperpolarizing current led to a significant decrease in fluorescence (Fig. 3c). When the same amount of depolarizing current was injected into the HSN cell, fluorescence increased in the $\mathrm{dCH}$ cell as well (Fig. $3 d$ ). Injection of hyperpolarizing current into the HSN cell led to a decreased fluorescence in the $\mathrm{dCH}$ cell (Fig. 3e). This parallels the results reported above with dual intracellular recording techniques and demonstrates the usefulness of calcium imaging for connectivity analysis.

To obtain information about the spatial aspects of the connectivity, we filled a $\mathrm{vCH}$ cell with the calcium indicator and recorded sequentially from two HS cells, an HSE cell and an HSS cell (Fig. 4a). When we depolarized the $\mathrm{vCH}$ cell directly by current injection into the $\mathrm{vCH}$ cell itself, a strong fluorescence increase was observed all over the cell, within the lobula plate arborizations, as well as the protocerebral ramifications (Fig. 4b). In contrast, when the depolarizing current was injected into the HSE cell, fluorescence increased only in the upper part of the lobula plate branches of the $\mathrm{vCH}$ cell (Fig. 4c). Depolarization of the HSS cell resulted in a fluorescence signal restricted to the lower part of the vCH cell dendrite (Fig. $4 d$ ). The strong dependence of the dendritic location of $\mathrm{vCH}$ cell fluorescence change on the exact type of HS cell into which the current was injected was further demonstrated when the fluorescence change was spatially averaged within the areas outlined in Figure 4, $c$ and $d$, 
a
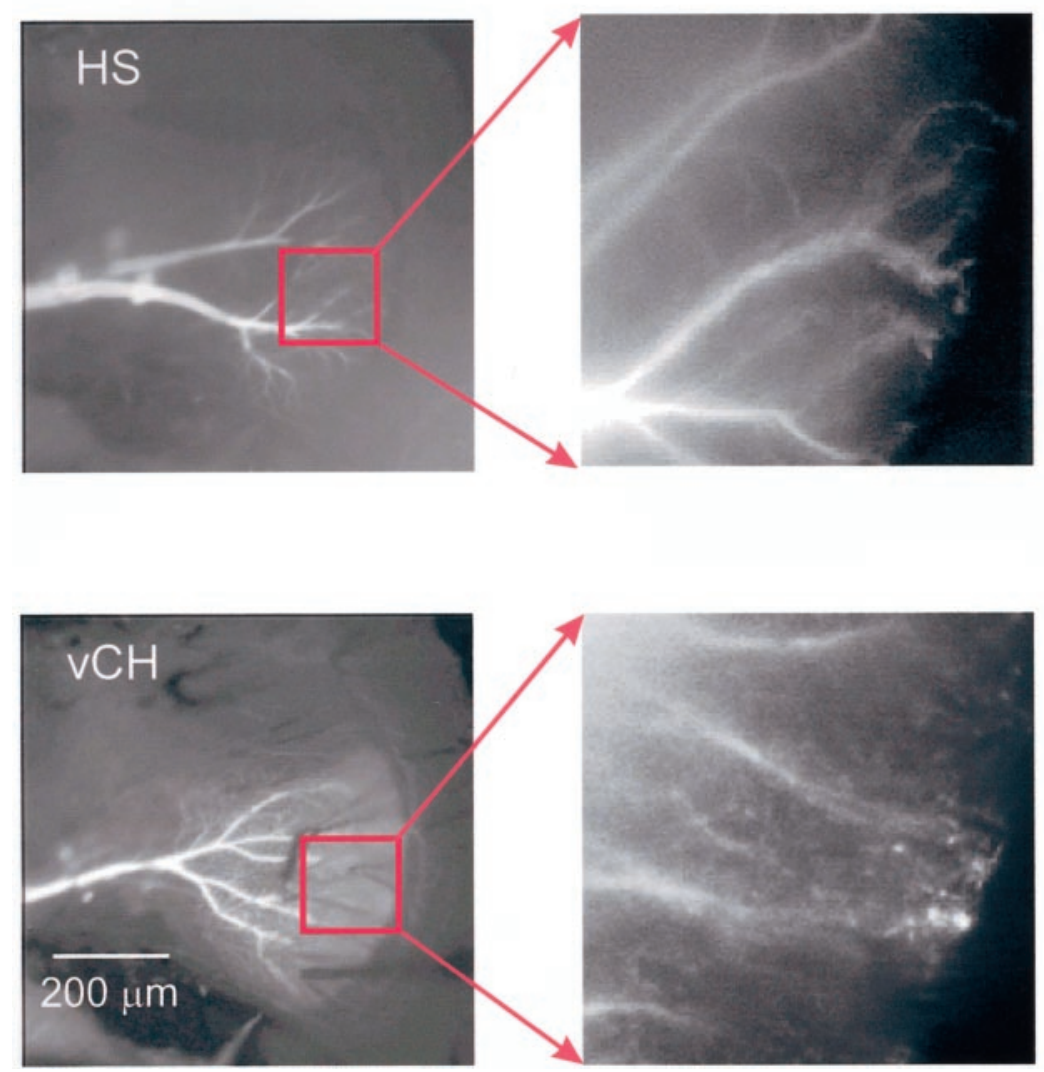

C
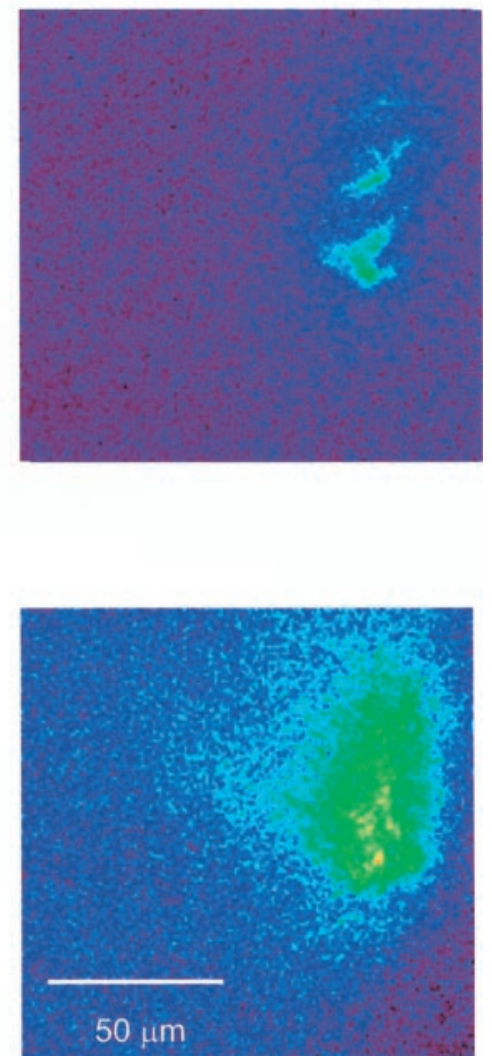

Figure 5. Small local motion stimuli lead to locally confined dendritic activity in both HS and CH cells. $a$, $b$, Raw fluorescence images showing the anatomy of the cells tested. The vCH cell was filled with Calcium Green, and the HSS cell was filled with Calcium Orange. $c$, False-color images of the relative fluorescence change in both cells after local visual motion stimulation $\left(12^{\circ} \times 12^{\circ}\right)$. A Zeiss Epiplan $10 \times, 0.20$ NA lens was used in the photographs of $a$, and a Zeiss Epiplan $40 \times, 0.75$ NA water immersion lens was used for the images in $b$ and $c$. Color scale identical to Figure 3 .

and displayed as a time course $(c, d$, right). These data speak in favor of a dendro-dendritic coupling between $\mathrm{HS}$ and $\mathrm{CH}$ cells.

Our finding of a dendro-dendritic coupling between HS and $\mathrm{CH}$ cells can explain the previous observation of retinotopic calcium accumulation in CH cell dendrites (Egelhaaf et al., 1993), even if $\mathrm{CH}$ cells did not receive direct synaptic input from local motion detectors. To investigate the consequences of this coupling on the visually evoked calcium signal in detail, we optically recorded simultaneously from $\mathrm{HS}$ and $\mathrm{CH}$ cells using two different calcium indicators, a short-wavelength dye (Calcium Green) in the $\mathrm{CH}$ cell and a long-wavelength dye (Calcium Orange) in the HS cell and presented a small local motion stimulus to the fly. As can be seen in Figure 5, the calcium signal in the $\mathrm{CH}$ cell was still local and comprised only a fraction of the area of overlap between both dendrites. However, the $\mathrm{CH}$ cell calcium signal appeared spatially blurred and enlarged compared with the signal in the HS cell dendrite (Fig. 5c, compare top and bottom rows). A quantitative evaluation of the data obtained in this and two other experiments revealed that, given a threshold of $50 \%$ of the maximum calcium signal, the dendritic area in HS cells activated by the local motion stimulus comprised only $8.7 \pm 1.8 \%$ (SEM) of the area activated simultaneously in $\mathrm{CH}$ cell dendrites. As a control, we also switched the dyes injecting Calcium Orange in a $\mathrm{CH}$ cell and Calcium Green in an HS cell. The result was again that the activated area in $\mathrm{CH}$ cells was substantially larger than the one in HS cells.
To discriminate between a chemical and an electrical coupling of $\mathrm{HS}$ and $\mathrm{CH}$ cells, we tried to block the effect of current injection into $\mathrm{HS}$ cells onto the calcium signal in $\mathrm{CH}$ cells. In brief, blocking agents of the nicotinic pathways, such as curare $(n=1)$, mecamylamine $(n=2)$, and $\alpha$-bungarotoxin $(n=1)$, all of which were effective in vitro (Brotz and Borst, 1996) and also eliminated the visual response in HS cells in our in vivo experiments, failed to disrupt the influence of HS current injection on $\mathrm{CH}$ cell potential (data not shown). However, the gap junction blocker Carbenoxolone (Wong et al., 1998) [2 $\mu$ l of $10 \mathrm{~mm}$ Carbenoxolone disodium salt (Sigma) in fly hemolymph] completely blocked the influence of HS current injection onto $\mathrm{CH}$ cells in three of three different experiments on various cell pair combinations of $\mathrm{HS}$ and $\mathrm{CH}$ cells. Although we cannot exclude other possible neurotransmitters at the moment, we conclude that the connection between HS and CH cells is not based on chemical transmission but rather through electrical synapses of the gap junction type. However, we never observed any kind of dye coupling between $\mathrm{HS}$ and $\mathrm{CH}$ cells, even when using small fluorescent probes such as calcein (Molecular Probes).

\section{DISCUSSION}

The above results show that $\mathrm{CH}$ cells receive synaptic input on the ipsilateral side from HS cells via dendro-dendritic electrical synapses. This particular kind of wiring between the dendrites of 
HS and $\mathrm{CH}$ cells has interesting implications for a couple of previous findings, which will be discussed in the following.

First of all, trying to fit passive and active membrane parameters of compartmental models from faithfully reconstructed $\mathrm{CH}$ cells to a large body of experimental data only led to unsatisfying results (Haag et al., 1999). In particular, the experimentally determined spatial integration properties of $\mathrm{CH}$ cells revealed a much stronger saturation characteristic than was predicted from simulations based on estimates of their dendritic membrane resistance. In the simulations, however, it was assumed that $\mathrm{CH}$ cells received direct and exclusive synaptic input from retinotopically arranged local motion detectors. As the above results demonstrate, this assumption turned out to be wrong. Our current modeling efforts on $\mathrm{CH}$ cells will show whether incorporating the new findings about the $\mathrm{HS}-\mathrm{CH}$ cell connectivity will eventually be able to resolve these previous inconsistencies.

Another puzzle applied to dendritic calcium measurements in other LPTCs and the finding of chemical output synapses in the dendrite of $\mathrm{CH}$ cells. As was shown in VS cells, preferred as well as null direction motion elicited a rise in dendritic calcium (Borst and Single, 2000; Single and Borst, 2002). This finding was explained by the low direction selectivity of the excitatory input, which presumably acts on LPTCs dendrites via calciumpermeable nicotinic acetylcholine receptors (Brotz and Borst, 1996; Oertner et al., 2001). If CH cells received the same kind of input from local motion-sensitive elements as VS cells, their dendritic transmitter release would substantially loose directionality, a problem that may have been overcome by coupling $\mathrm{CH}$ cell dendrites electrically to HS cells. Consistent with this kind of connectivity, $\mathrm{CH}$ cells indeed do not show an increase but rather a decrease in dendritic calcium during null direction motion (Single, 1998), as if the visually induced dendritic calcium concentration followed the membrane potential solely dictated by a voltage-activated calcium current (Haag and Borst, 2000).

An important aspect of the dendro-dendritic $\mathrm{HS}-\mathrm{CH}$ cell coupling might relate to the spatial blur of motion information in the $\mathrm{CH}$ cell dendrite compared with the $\mathrm{HS}$ cell dendrite. $\mathrm{CH}$ cells have been implied to be inhibitory onto a class of LPTCs selectively sensitive to small-field or relative motion (Egelhaaf, 1985b; Gauck and Borst, 1999; Kimmerle and Egelhaaf, 2000b). When $\mathrm{CH}$ cells were laser ablated, small-field selective cells lost much of their response selectivity, now responding stronger to a large-field stimulus than to a small moving object (Warzecha et al., 1993). Assuming that these cells receive a motion image like HS cells on their dendrite, dendro-dendritic inhibition by $\mathrm{CH}$ cells would be analogous to subtracting a blurred image from an original one, resulting in an enhancement of motion discontinuities. In case of homogeneous large-field motion input, this operation would result in a rather complete cancellation of the excitatory by the inhibitory input, whereas relative motion cues, such as occurring by either object motion or by self motion in a three-dimensional environment, would lead to an contrast enhancement at the motion edges, just like conventional high-pass filtering in image processing. However, whether the sensitivity of small-field-selective neurons in the fly lobula plate indeed relies critically on the blurring of motion input in the $\mathrm{CH}$ cell dendrite remains to be validated in future experiments.

Beside the functional implications of the dendro-dendritic coupling between $\mathrm{HS}$ and $\mathrm{CH}$ cells for the response properties of $\mathrm{CH}$ cells, the above experiments also demonstrate that, in HS cells of the fly visual system, the dendrites act in different ways depending on what output neuron is considered: whereas for the neuron postsynaptic to the axon terminal all positional information is lost after spatial integration, the partner cell postsynaptic to the dendrite receives spatially resolved signals corresponding to the location of where in visual space motion stimuli are occurring. The present data, thus, add a new level of complexity to the processing capabilities of single nerve cells (for review, see Hausser et al., 2000; Segev and London, 2000), showing that, at least in the case considered here, the function of the dendrite of a neuron cannot be described in isolation but rather depends on which postsynaptic partner cell is being considered.

\section{REFERENCES}

Borst A, Egelhaaf M (1992) In vivo imaging of calcium accumulation in fly interneurons as elicited by visual motion stimulation. Proc Natl Acad Sci USA 89:4139-4143.

Borst A, Haag J (1996) The intrinsic electrophysiological characteristics of fly lobula plate tangential cells. I. Passive membrane properties. J Comput Neurosci 3:313-336.

Borst A, Single S (2000) Local current spread in electrically compact neurons of the fly. Neurosci Lett 285:123-126.

Brotz TM, Borst A (1996) Cholinergic and GABAergic receptors on fly tangential cells and their role in visual motion detection. $J$ Neurophysiol 76:1786-1799.

Chan WP, Prete F, Dickinson MH (1998) Visual input to the efferent control system of a fly's "gyroscope." Science 280:289-292.

Eckert H, Dvorak DR (1983) The centrifugal horizontal cells in the lobula plate of the blowfly Phaenicia sericata. J Insect Physiol 29:547-560.

Egelhaaf M (1985a) On the neuronal basis of figure-ground discrimination by relative motion in the visual system of the fly. I. Behavioural constraints imposed on the neuronal network and the role of the optomotor system. Biol Cybern 52:123-140.

Egelhaaf M (1985b) On the neuronal basis of figure-ground discrimination by relative motion in the visual system of the fly. II. Figuredetection cells, a new class of visual interneurones. Biol Cybern $52: 195-209$

Egelhaaf M, Borst A, Warzecha AK, Flecks S, Wildemann A (1993) Neural circuit tuning fly visual neurons to motion of small objects. II. Input organization of inhibitory circuit elements revealed by electrophysiological and optical recording techniques. J Neurophysiol 69:340-351.

Gauck V, Borst A (1999) Spatial response properties of contralateral inhibitioned lobula plate tangential cells in the fly visual system. J Comp Neurol 406:51-71.

Gauck V, Egelhaaf M, Borst A (1997) Synapse distribution on VCH, an inhibitory, motion-sensitive interneuron in the fly visual system. J Comp Neurol 381:489-499.

Gilbert C, Gronenberg W, Strausfeld NJ (1995) Oculomotor control in calliphorid flies: head movements during activation and inhibition of neck motor neurons corroborate neuroanatomical predictions. J Comp Neurol 361:285-297.

Gronenberg W, Strausfeld NJ (1990) Descending neurons supplying the neck and flight motor of diptera: physiological and anatomical characteristics. J Comp Neurol 302:973-991.

Haag J, Borst A (1996) Amplification of high-frequency synaptic inputs by active dendritic membrane processes. Nature 379:639-641.

Haag J, Borst A (2000) Spatial distribution and characteristics of voltage-gated calcium signals within visual interneurons. J Neurophysiol 83:1039-1051.

Haag J, Borst A (2001) Recurrent network interactions underlying flowfield selectivity of visual interneurons. J Neurosci 21:5685-5692.

Haag J, Theunissen F, Borst A (1997) The intrinsic electrophysiological characteristics of fly lobula plate tangential cells. II. Active membrane properties. J Comput Neurosci 4:349-369.

Haag J, Vermeulen A, Borst A (1999) The intrinsic electrophysiological characteristics of fly lobula plate tangential cells. III. Visual response properties. J Comput Neurosci 7:213-234.

Hausen K (1982) Motion sensitive interneurons in the optomotor system of the fly. I. The horizontal cells: structure and signals. Biol Cybern 45:143-156.

Hausen K (1984) The lobula-complex of the fly: structure, function and significance in visual behaviour. In: Photoreception and vision in invertebrates (Ali MA, ed), pp 523-559. New York: Plenum.

Hausen K, Wolburg-Buchholz K, Ribi WA (1980) The synaptic organization of visual interneurons in the lobula complex of flies. Cell Tissue Res 208:371-387.

Hausser M, Spruston N, Stuart GJ (2000) Diversity and dynamics of dendritic signaling. Science 290:739-744.

Heisenberg M, Wolf R (1979) On the structure of yaw torque in visual 
flight orientation of Drosophila melanogaster. J Comp Physiol 130:113-130.

Hengstenberg R (1977) Spike response of "non-spiking” visual interneurone. Nature 270:338-340.

Hengstenberg R, Hausen K, Hengstenberg B (1982) The number and structure of giant vertical cells (VS) in the lobula plate of the blowfly Calliphora erytrocephala. J Comp Physiol [A] 149:163-177.

Kimmerle B, Egelhaaf M (2000a) Detection of object motion by a fly neuron during simulated flight. J Comp Physiol [A] 186:21-31.

Kimmerle B, Egelhaaf M (2000b) Performance of fly visual interneurons during object fixation. J Neurosci 20:6256-6266.

Meyer EP, Matute C, Streit P, Nässel DR (1986) Insect optic lobe neurons identifiable with monoclonal antibodies to GABA. Histochemistry $84: 207-216$

Oertner TG, Brotz T, Borst A (2001) Mechanisms of dendritic calcium signaling in fly neurons. J Neurophysiol 85:439-447.

Reichardt W, Poggio T (1979) Figure-ground discrimination by relative movement in the visual system of the fly. I. Experimental results. Biol Cybern 35:81-100.

Segev I, London M (2000) Untangling dendrites with quantitative models. Science 290:744-750.

Single S (1998) Mechanismen und Funktion dendritischer Kalziumsignale in visuellen Interneuronen der Fliege. $\mathrm{PhD}$ thesis, University of Tuebingen.

Single S, Borst A (1998) Dendritic integration and its role in computing image velocity. Science 281:1848-1850.

Single S, Borst A (2002) Different mechanisms of calcium entry within different dendritic compartments. J Neurophysiol 87:1616-1624.

Warzecha AK, Egelhaaf M, Borst A (1993) Neural circuit tuning fly visual interneurons to motion of small objects. I. Dissection of the circuit by pharmacological and photoinactivation techniques. J Neurophysiol 69:329-339.

Wong WT, Sanes JR, Wong ROL (1998) Developmentally regulated spontaneous activity in the embryonic chick retina. J Neurosci 18:88398852 . 